\title{
Are emojis processed like words?: Eye movements reveal the time course of semantic processing for emojified text
}

\author{
Eliza Barach ${ }^{1}$ (D) Laurie Beth Feldman ${ }^{1} \cdot$ Heather Sheridan ${ }^{1}$
}

Accepted: 11 December 2020 / Published online: 28 January 2021

(C) The Psychonomic Society, Inc. 2021

\begin{abstract}
Emojis have many functions that support reading. Most obviously, they convey semantic information and support reading comprehension (Lo, CyberPsychology \& Behavior, 11 [5], 595-597, 2008; Riordan, Computers in Human Behavior, 76, 75$86,2017 b)$. However, it is undetermined whether emojis recruit the same perceptual and cognitive processes for identification and integration during reading as do words. To investigate whether emojis are processed like words, we used eye tracking to examine the time course of semantic processing of emojis during reading. Materials consisted of sentences containing a target word (e.g., coffee in the sentence "My tall coffee is just the right temperature") when there was no emoji present and when there was a semantically congruent (i.e., synonymous) emoji (e.g., the cup of coffee emoji, - ) or an incongruent emoji (e.g., the beer mug emoji, [D) present at the end of the sentence. Similar to congruency effects with words, congruent emojis were fixated for shorter periods and were less likely to be refixated than were incongruent emojis. In addition, congruent emojis were more frequently skipped than incongruent emojis, which suggests that semantic aspects of emoji processing begin in the parafovea. Finally, the presence of an emoji, relative to its absence increased target-word skipping rates and reduced total time on target words. We discuss the implications of our findings for models of eye-movement control during reading.
\end{abstract}

Keywords Emojis $\cdot$ Eye movements and reading $\cdot$ Reading models $\cdot$ Parafoveal processing

Are emojis processed like words? As with words, a core function of emojis is to convey semantic information (Lo, 2008; Riordan, 2017b). Although emojis serve many different functions to support reading, it is unknown whether the same cognitive and perceptual processes that support the identification and integration of words during reading also extend to emojis. Given the growing popularity of emojis on the internet, social media (Novak, Smailović, Sluban, \& Mozetič, 2015), and in text-based communication (Tigwell \& Flatla, 2016; i.e., more than 3,000 emojis are available to date; Unicode 12.1; https:// unicode.org/emoji/charts/full-emoji-list.html), we aimed to determine whether the time course of semantic processing for emojis is similar to words. The time course of semantic processing provides empirical constraints for the development of models of eye movement control during reading (for reviews, see Rayner, 1998, 2009). Considering the ubiquity

Eliza Barach

ebarach@albany.edu

1 Department of Psychology, University at Albany, State University of New York, 1400 Washington Avenue, Albany, NY 12222, USA of emojis, their inclusion in these models will provide a more comprehensive understanding of reading under ecologically valid conditions. Towards this goal, we used eye tracking to examine the time course of semantic processing of emojis that accompanied text during natural sentence reading. Accordingly, we will briefly summarize prior work on the semantic processing of both words and emojis, and we will then introduce the present study's objectives and predictions.

To delineate the time course of semantic processing, a large body of work has manipulated the extent to which target words are semantically congruent versus incongruent with prior sentence contexts (i.e., semantic congruency). Semantic processing can have both an early and a longlasting effect on target word processing, as indexed by effects on both early and late eye-tracking measures (for reviews, see Rayner, 1998, 2009), and by event-related potential (ERP) effects on the N1 component (e.g., Penolazzi, Hauk, \& Pulvermüller, 2007; Sereno, Brewer, \& O’Donnell, 2003), and the later N400 component (e.g., Dimigen, Kliegl, \& Sommer, 2012; Kutas \& Hillyard, 1980).

Evidence that the semantic processing of words can begin in the parafovea (i.e., before a word is directly fixated) derives 
from studies that used the gaze-contingent boundary paradigm (Rayner, 1975) to replace preview words (i.e., upcoming words in the parafovea) with a target word during a saccade towards the preview word. This paradigm revealed that reading is facilitated when the preview is semantically congruent with the target (i.e., semantic preview benefits; Hohenstein \& Kliegl, 2014; Rayner \& Schotter, 2014; Schotter, 2013; Schotter, Lee, Reiderman, \& Rayner, 2015), but more specifically when it is congruent with the sentence context (i.e., plausibility preview benefits; Schotter \& Jia, 2016; Veldre \& Andrews, 2016, 2017). Intriguingly, semantic preview benefits have typically been more reliable for text in Chinese relative to text in alphabetic languages (e.g., Yan, Richter, Shu, \& Kliegl, 2009; Yan, Zhou, Shu, \& Kliegl, 2012; Yang, Wang, Tong, \& Rayner, 2012). As discussed by Schotter, Angele, and Rayner (2012), one possible explanation for this crosslanguage difference is that the written form of Chinese words is spatially more compact than words in alphabetic languages. This compact layout could facilitate parafoveal processing by positioning more of the form of upcoming words closer to the fovea. Similar to Chinese characters, emojis also convey rich semantic information in a compact spatial layout. Thus, emojis seem ideal for exploring the perceptual and cognitive limits of the effect of semantic processing on eye movement control during reading.

Although the time course of semantic processing for words has been extensively investigated, much less is known about the processing of emojis. Prior investigations of emoji processing have used a substitution approach whereby emojis substituted for specific words in a sentence (e.g., the pizza and smiley face emojis could replace the words pizza and happy, respectively). Using this substitution approach with the self-paced reading paradigm (Cohn, Roijackers, Schaap, \& Engelen, 2018) and the rapid serial visual presentation (RSVP) paradigm (Tang, Chen, Zhao, \& Zhao, 2020; Weissman, 2019), results have demonstrated that emojis that are semantically congruent with the word they replace are read faster (Cohn et al., 2018) and elicit reduced N400 amplitudes (Tang et al., 2020; Weissman, 2019), compared with emojis that are semantically incongruent with the word they replace. Akin to the N400 effect with words (e.g., Dimigen et al., 2012; Kutas \& Hillyard, 1980), the reduction in N400 amplitudes for congruent compared with incongruent emojis suggests that emojis are integrated with text in a similar manner as are words (Weissman, 2019).

While these prior findings offer a glimpse into the online processing of emojis, details about their time course remain unclear. In particular, it is unclear how early the semantic processing of emojis begins during natural reading, as well as the extent to which emoji processing can begin in the parafovea. To address these questions, we tested whether semantic congruency can rapidly modulate the eye-movement record for emojis, as was previously shown for words. In doing so, we build on a large prior literature that has used eye tracking to provide fine-grained time-course processing about word processing during reading (for reviews, see Rayner, 1998, 2009). Extending this literature, we aimed to use emojis to assess the generalizability of the assumptions of contemporary models of eye movement control, such as $E-Z$ Reader (Reichle, Pollatsek, Fisher, \& Rayner, 1998; Reichle, Pollatsek, \& Rayner, 2012) and SWIFT (Engbert, Longtin, \& Kliegl, 2002; Engbert, Nuthmann, Richter, \& Kliegl, 2005). Of particular relevance to our study, one of the longstanding controversies among models of eye movement during reading concerns the extent to which lexical and linguistic variables, such as semantic congruency, can rapidly modulate fixation durations from moment-to-moment during reading (i.e., the direct cognitive control hypothesis). As reviewed elsewhere (e.g., Reingold, Sheridan, \& Reichle, 2015), some models adopt the assumption of direct cognitive control of eye movements by high-level variables, whereas other models assume that the online control of fixation durations is instead primarily driven by lower level visual and/or oculomotor variables. To the extent that we find that semantic congruency can rapidly impact the eye-movement record for emojis, our study would provide further evidence for the direct cognitive control hypothesis in a new type of naturalistic reading setting (i.e., emojified text). It is unknown to what extent the assumptions of current models of eye-movement control will extend to emojis, given that emojis differ from words in alphabetic languages due to their compact visual format, and because emojified text entails the integration of verbal information (i.e., text) with nonverbal information (i.e., emojis). Thus, by examining the extent to which the time course of emoji processing is "word-like," we aimed to explore the generalizability of the assumptions of current models of eye-movement control during reading.

We also extend prior emoji research that has largely focused on emoji-word substitutions (Cohn et al., 2018; Gustafsson, 2017; Tang et al., 2020; Weissman, 2019) by instead presenting the text and the emojis in our study at the same time (e.g., the apple emoji accompanied the word apple in the sentence). Although emojis commonly replace a word in text, they are also commonly appended to the end of the message (Cramer, Juan, \& Tetreault, 2016). Concurrent presentation of text and emoji allowed us to study how the verbal information conveyed by text interacts with the nonverbal information conveyed by the emojis. Finally, as an additional methodological refinement, our design allowed us to contrast the same sentences (they served as their own controls) with and without emojis, as well as in the presence of semantically congruent and incongruent emojis.

To summarize, we used eye tracking to document the time course of semantic processing of emojis during natural sentence reading. We presented sentences containing target words (e.g., coffee in the sentence "My tall coffee is just the 
right temperature") in three different conditions: (1) the synonymous condition, in which the sentence ended with an emoji that has the same meaning as the target word (e.g., the coffee cup emoji - ); (2) the incongruent condition, in which the sentence ended with an emoji that did not depict the target word (e.g., the beer mug emoji [D); and (3) the no-emoji condition, in which the sentence did not contain an emoji. We selected emojis that could replace target words and maintain sentence meaning (i.e., "emoji synonyms") in the synonymous condition so as to create a condition of strong semantic congruency, thereby building on prior work that used synonyms to probe for semantic preview benefits during reading (Schotter, 2013).

Whereas prior work on emoji processing has largely relied on self-reports (e.g., Kelly \& Watts, 2015; Riordan, 2017a, 2017b), self-paced reading paradigms (e.g., Cohn et al., 2018), modified RSVP paradigms (e.g., Weissman, 2019) and overall reading times (e.g., Gustafsson, 2017), we obtained fine-grained time-course information by analyzing both early and late eye-movement measures of emoji processing. We predicted reduced processing for congruent (i.e., synonymous) relative to incongruent emojis. Under the premise that emojis and words are processed similarly (Weissman, 2019), and given the breadth of prior work documenting both early and late word congruency effects (Dimigen et al., 2012; Kutas \& Hillyard, 1980; Penolazzi et al., 2007; Rayner, 1998, 2009; Sereno et al., 2003), we expected the effect of emoji congruency to emerge in early measures (i.e., first-pass fixation measures and skipping rates) and to persist into later measures (i.e., total time). Likewise, we expected reduced processing of target words that were accompanied by congruent emojis, particularly for later measures (e.g., total time on the target word) that include regressions back to the target word. More specifically, we expected this congruency effect on the target word to be particularly salient for late measures because conditions of congruency were established by the emoji that always occurred after the target word in the sentence. Finally, based on the previous studies with emojified text (Cohn et al., 2018; Gustafsson, 2017), we anticipated longer overall sentence reading times for sentences with emojis relative to sentences without emojis.

\section{Method}

\section{Participants}

Sixty native-English-speaking undergraduate students (39 females, 21 males, $M_{\text {age }}=19$ years) with normal or corrected-tonormal vision were recruited from the SUNY Albany SONA system. All participants reported owning a smartphone, with 55 having Apple and five having Android mobile operating systems (MOS). The average age at which participants received their first smartphone was 12.9 years and their average reported daily usage was 5.3 hours. All participants reported using emojis. Since emojis have different renderings across platforms and our stimuli were rendered in Apple IOS, we removed the five participants who reported having Android MOS. Our final sample size consisted of 55 participants and was determined a priori based on previous studies in the reading and eye-movements literature (for reviews, see Rayner, 1998, 2009). We selected a large sample size because it was difficult to predict the effect sizes that we would observe given the novelty of our approach.

\section{Materials and design}

There were 89 experimental sentences (see Table 5 in the Appendix A) that ranged in length from five to 13 words ( $M$ $=8.99$ words, excluding the emoji). ${ }^{1}$ Each sentence contained a target word that ranged in length from four to 13 letters $(M=$ 6.4), with a mean word frequency of 25.77 words per million (SUBLETX; Brysbaert \& New, 2009). Each sentence appeared on a single line, and the target word could appear in any location in the sentence, with the exception of the first or last word.

The 89 experimental sentences were displayed in three conditions: (1) The synonymous condition contained an emoji that was semantically congruent with the target word (e.g., Homemade cookies are delicious : $:$ ); (2) the incongruent condition contained an emoji that was semantically incongruent with the target word (e.g., Homemade cookies are delicious 9$)^{2}$ and the no-emoji condition did not contain an emoji (e.g., Homemade cookies are delicious). The 178 emojis in our study (see Table 5 in Appendix A) were from Apple IOS 12.2. In the synonymous and incongruent conditions, the emoji was always located immediately after the last word in the sentence, with a minimum of two words and maximum of eight words between the emoji and the target word $(M=4.8$

\footnotetext{
${ }^{1}$ We excluded the following additional item from our analyses: "There was a lot of anger/emotion after exam grades were posted 30 . (3)." We decided to remove this item to restrict the semantic congruity analyses to object emojis, given the preliminary evidence that not all emojis contribute to a message in the same manner (Barach et al., 2020; Na'aman et al., 2017).

${ }^{2}$ The congruity between emoji label and target word was established by consulting the emojipedia database (https://emojipedia.org). Of the 89 "synonymous" emojis (i.e., the emojis that were congruent with the target words), only the target words SEDAN, FLIES, TYPIST, MOBILE PHONE, and BEEF were not listed as labels in emojipedia for the congruent emojis AUTOMOBILE, AIRPLANE, KEYBOARD, TEXT, and STEAK, respectively. In five other cases, the emoji label was a morphological relative of the target word, such as the emoji of SKIER for the target word SKIING and WIND for WINDY. Thus, approximately $90 \%$ of the emojitarget word pairs were congruent according to the labels in the emojipedia database. For the incongruent emojis, the labels in the database never matched with the target word. In addition, five independent raters reviewed the stimuli to confirm that the selected emojis were easily recognizable and were unequivocally semantically congruent or incongruent with their respective sentence/ target word.
} 
(a) My tall coffee is just the right temperature

(b) My tall coffee is just the right temperature

(c) My tall coffee is just the right temperature

Fig. 1 Example of the three sentence types in which the sentence contained a (a) congruent emoji, (b) incongruent emoji, and (c) No emoji. Approximately 1 degree of visual angle was equivalent to 3.21 letter characters and 1.69 emoji characters

intervening words). We counterbalanced the assignment of sentences to conditions such that across participants each sentence occurred equally often in each of the three conditions (synonymous, incongruent, no emoji), but each participant saw only one version of each sentence.

To confirm that the emojis in the synonymous and incongruent conditions were equated for processing difficulty, an additional 30 participants read sentences that contained a general target word that fit semantically with either emoji (see Table 5 in Appendix A). There were no differences between emoji processing across all measures with the general target words (see Appendix Table 7).

\section{Apparatus and procedure}

Eye movements were measured with an SR Research EyeLink 1000 Plus system with a sampling rate of $1000 \mathrm{~Hz}$. Viewing was binocular, but only the right eye was monitored. A chin rest and forehead rest were used to minimize head movements. Following calibration, average gaze-position error was less than $0.5^{\circ}$. The sentences were presented on a $24-$ inch Asus VG248QE monitor, with a refresh rate of $144 \mathrm{~Hz}$ and a screen resolution of $1,920 \times 1,080$ pixels. All letters were lowercase (except where capitals were appropriate) and were shown in single-spaced, 22-point black Courier New font on a white background. The emoji was presented in 28pt font. Participants were seated $92 \mathrm{~cm}$ from the monitor. Approximately 1 degree of visual angle was equivalent to 3.21 letter characters and 1.69 emoji characters (see Fig. 1 for an example trial).

Participants were instructed to read for comprehension. They initiated each trial by pressing a button while fixating on a cross on the left side of the screen, and they ended each trial by pressing a button. To ensure that participants read for comprehension, 30 additional filler sentences (approximately $25 \%$ of trials) were followed by a comprehension question. To ensure participants were processing both the emoji and the sentence we included two types of comprehension questions that queried about the sentence content or the emoji specifically (see Table 6 in Appendix A). Average comprehension accuracy was $97 \%$. The order of sentences was randomized, with a new random order for each participant.

\section{Results}

We analyzed the data using the lme4 package (Version 1.1.12; Bates, Mächler, Bolker, \& Walker, 2015) within the R studio environment to run generalized linear mixed-effects models (LMMs). For each model, emoji congruency was entered as a fixed effect, and subjects and items were treated as random effects.

To discover the optimal model a full model using the maximal random effects structure (Barr, Levy, Scheepers, \& Tily, 2013) was first fitted. If a model failed to converge it was systematically trimmed until it converged, by removing correlations between random effects, and then random effects associated with the smallest variance. Once models converged we used restricted maximum likelihood (REML) estimates for model comparisons involving correlation parameters or variance components (see Appendix Table 8 for a summary of random effects structures). Patterns of significance were the same for the log-transformed data as for the untransformed data, so we report the analysis of the untransformed data to maintain transparency of the effect sizes.

Linear mixed-effects regressions were used for fixation duration measures and sentence reading time and we report the regression coefficients which represent the effect size (in milliseconds) of the reported comparison as well as its associated $t$ value. Logistic mixed-effects regressions were used for

Fig. 2 Means (aggregated by subject) for the eye movement measures and sentence reading time. Specifically, we analyzed eye movements (a: first fixation; b: single fixation; c: gaze duration; d: total time; e: probability of single fixations; f: probability of skipping) for the emoji as a function of emoji congruency (synonymous vs. incongruent) as well as for the target word as a function of emoji condition (synonymous vs. incongruent vs. no emoji). In addition, we analyzed sentence reading time (f) as a function of emoji condition (synonymous vs. incongruent vs. noemoji). The error bars depict the standard error of the mean 
(a) First-Fixation

- Synonymous $\square$ Incongruent $\square$ No-Emoji

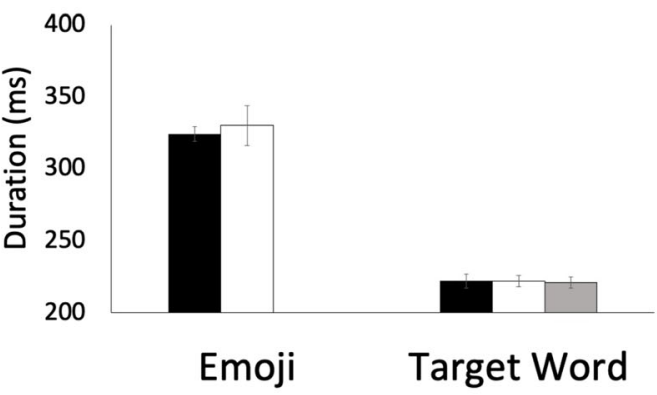

(c) Gaze

Synonymous $\square$ Incongruent $\square$ No-Emoji

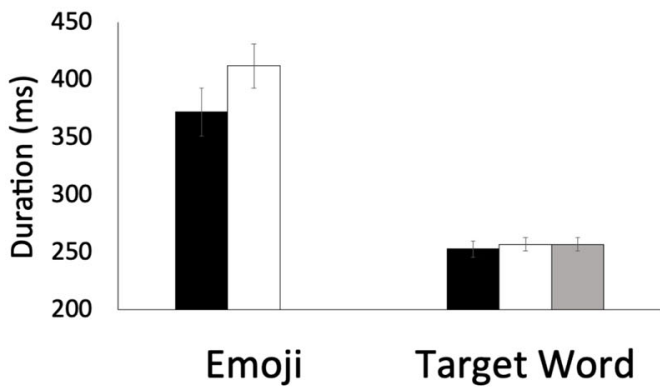

(e) Probability of Single-Fixations

- Synonymous $\square$ Incongruent $\square$ No-Emoji

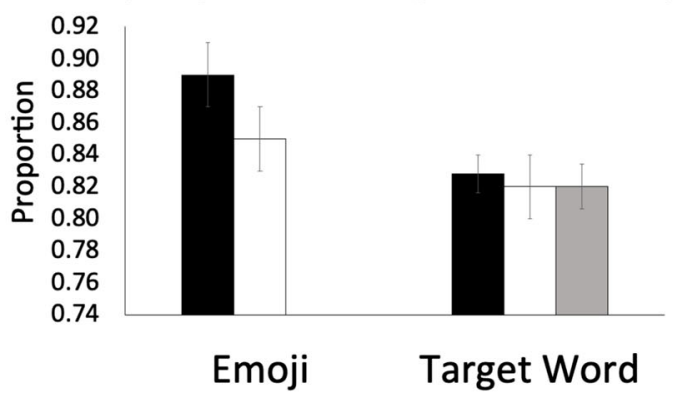

(b)

Single-Fixation

- Synonymous $\square$ Incongruent $\square$ No-Emoji

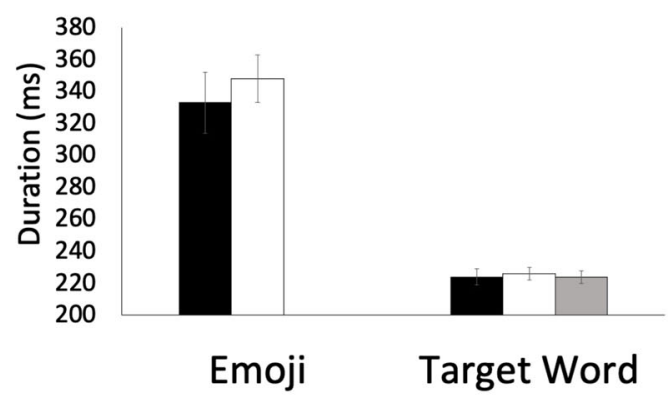

(d)

Total Time

$\square$ Synonymous $\square$ Incongruent $\square$ No-Emoji

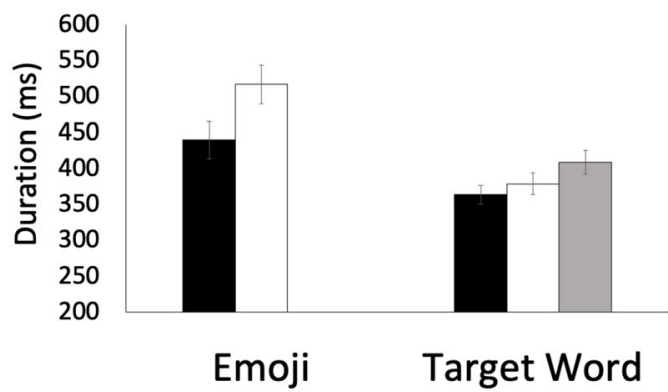

(f)

Probability of Skipping

- Synonymous $\square$ Incongruent $\square$ No-Emoji

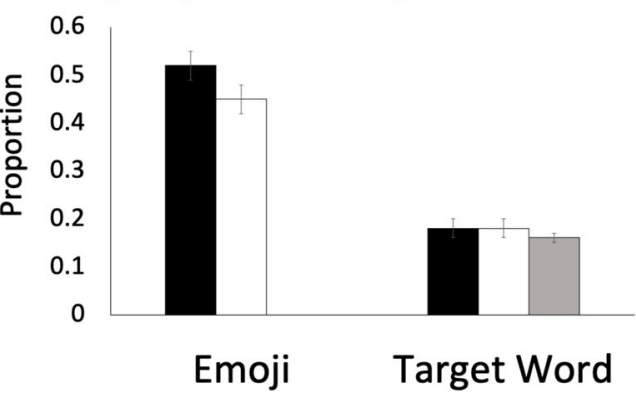

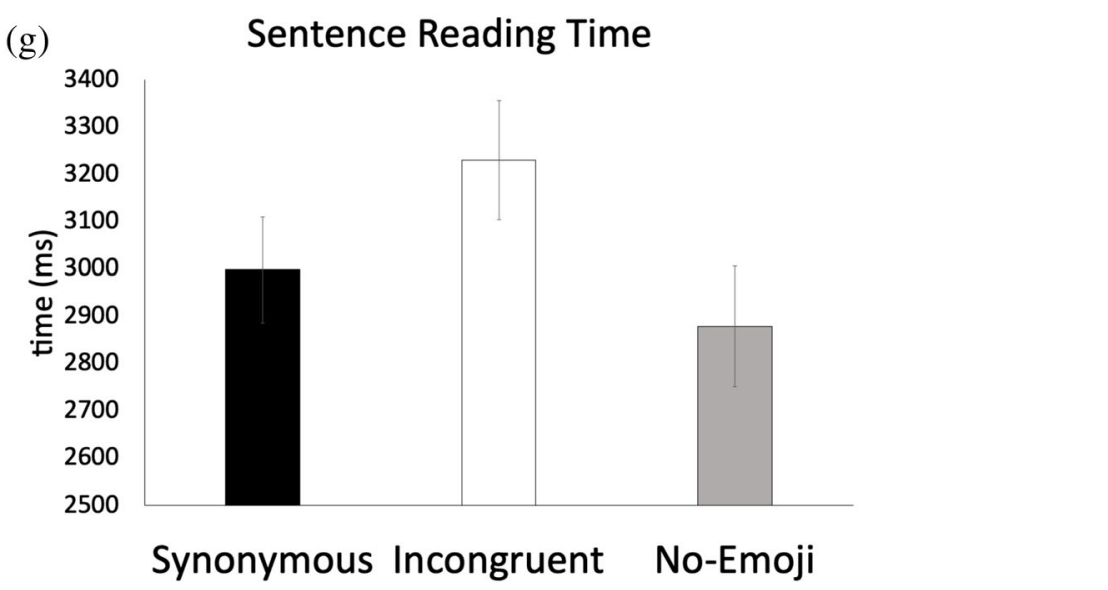


binary variables (probability of single-fixations and probability of skipping), and we report the regression coefficients which represent the effect size in log-odds space, as well as the $z$ and $p$ value of the effect coefficient. Absolute values of the $t$ and $z$ statistics greater than or equal to 1.96 indicate a significant effect at approximately the .05 alpha level.

\section{Overview of eye-tracking measures}

To examine the processing of the emojis and the target words, we analyzed the following eye-tracking measures: (1) first-fixation duration (i.e., the duration of the very first fixation on a target); (2) single-fixation duration (i.e., the first-fixation duration for the subset of trials in which there was only one firstpass fixation on the target); (3) gaze duration (i.e., the sum of all consecutive first-pass fixations on the target, prior to a saccade to another word); (4) total time (i.e., the sum of all the fixations on the target, including regressions back to the target); (5) probability of skipping (i.e., the proportion of trials in which there was no first-pass fixation on the target, regardless of whether or not the target was fixated later in the trial); and (6) probability of a single fixation (i.e., the proportion of trials in which there was only a single first-pass fixation on the target). Fixations that were shorter than $80 \mathrm{~ms}$ or more extreme than three standard deviations from each subject's mean on the emoji (1.7\% of trials) or the target word (2.6\% of trials) were excluded from fixation-based analyses (i.e., first fixation, single fixation, gaze, total time, probability of a single fixation).

\section{Emoji processing}

To investigate the time course of emoji processing, LMMs were built with one planned contrast to test the difference between synonymous and incongruent emojis. This contrast was achieved by setting the synonymous condition to the baseline (intercept) in the model and using the default contrast for the comparison of the synonymous condition to the incongruent

Table 1 Results of the linear mixed-effects models for the emoji for the fixation time measures (first fixation, single fixation, gaze duration, and total time) as a function of emoji congruency (synonymous vs. incongruent)

\begin{tabular}{llll}
\hline Measure & $b$ & $S E$ & $t$ \\
\hline First-fixation duration & $\mathbf{2 5 . 6 9}$ & $\mathbf{1 1 . 3 3}$ & $\mathbf{2 . 2 7}$ \\
Single-fixation duration & $\mathbf{3 6 . 5 2}$ & $\mathbf{1 2 . 3 2}$ & $\mathbf{2 . 9 6}$ \\
Gaze duration & $\mathbf{5 9 . 0 1}$ & $\mathbf{1 6 . 5 3}$ & $\mathbf{3 . 5 7}$ \\
Total time & $\mathbf{1 0 3 . 7 7}$ & $\mathbf{2 1 . 5 0}$ & $\mathbf{4 . 8 3}$ \\
\hline
\end{tabular}

Note. The regression coefficients that represent effect size in milliseconds reflect the absolute difference in processing between the synonymous condition (intercept) and the incongruent condition. Significant effects are indicated in boldface condition. For the fixation-based measures (i.e., first fixation, single fixation, gaze duration, total time, probability of a single fixation), trials were excluded from the analysis if readers skipped the emoji (48.5\%) or if there was a blink before or after the emoji (10.7\%). Means are depicted in Fig. 2.

As shown in Table 1, synonymous emojis exhibited faster processing relative to incongruent emojis, as revealed by shorter first-fixation durations $(b=25.69, t=$ 2.27), single-fixation durations $(b=36.52, t=2.96)$, gaze durations $(b=59.01, t=3.57)$, and shorter total times on the emoji ( $b=103.77, t=4.83$ ). There was also an increase in the proportion of single-fixations for synonymous emojis compared with incongruent emojis as shown in Table $2(z=-2.53, p<.05)$. As evidence that emoji processing may begin in the parafovea, skipping was higher for synonymous than for incongruent emojis $(z=$ $-4.74, p<.001$; see Table 2).

\section{Target word and sentence processing}

To examine how emojis (i.e., non-verbal elements) interact with text (i.e., verbal elements) during natural reading, target word LMMs were built with two planned contrasts (Helmert) such that the intercept corresponded to the grand mean: The first tested for a difference in processing between target words followed by synonymous versus incongruent emojis (i.e., a congruency effect), and the second tested for a difference in processing between the target words followed by an emoji (i.e., the mean of the synonymous and incongruent conditions) versus followed by no emoji. For the fixation based measures (i.e., first fixation, single fixation, gaze duration, total time, probability of a single fixation), trials were excluded if readers skipped the target word (17.2\%) or if there was a blink before or after the target word (6.8\%). Means are depicted in Fig. 2.

With the exception of the probability of skipping, early eye-tracking measures of target-word processing (i.e., first fixation, single fixation, gaze duration, probability of single

Table 2 Results of the linear mixed-effects models for the emoji for the fixation probability measures (probability of single fixations, probability of skipping) as a function of emoji congruency (synonymous vs. incongruent)

\begin{tabular}{lllll}
\hline Measure & $b$ & $S E$ & $z$ & $p$ \\
\hline Probability of Single-Fixations & -.41 & $\mathbf{. 1 6}$ & $-\mathbf{2 . 5 3}$ & $<.05$ \\
Probability of Skipping &.- .37 & $\mathbf{. 0 8}$ & $-\mathbf{4 . 7 4}$ & $<.001$ \\
\hline
\end{tabular}

Note. The regression coefficients which represent effect size in log-odds space reflect the difference in processing between the synonymous condition (intercept) and the incongruent condition. Significant effects are indicated in boldface. Negative $z$ s indicate a reduction in the effect, such that the log odds were reduced for the incongruent condition relative to the synonymous condition 
Table 3 Results of the linear mixed-effects models for the target word for the fixation time measures (first fixation, single fixation, gaze duration, and total time) as well as sentence reading times as a function of emoji congruency (synonymous, incongruent, no emoji)

\begin{tabular}{|c|c|c|c|c|}
\hline Measure & Contrast & $b$ & SE & $t$ \\
\hline \multirow[t]{3}{*}{ First-fixation duration } & Intercept & 221.34 & 4.06 & 54.54 \\
\hline & Synonymous vs. Incongruent & -.79 & 1.33 & -.60 \\
\hline & Emoji vs. No Emoji & -.19 & .76 & -.25 \\
\hline \multirow[t]{3}{*}{ Single-fixation duration } & Intercept & 223.99 & 4.29 & 52.20 \\
\hline & Synonymous vs. Incongruent & .28 & 1.49 & .19 \\
\hline & Emoji vs. No Emoji & -.08 & .86 & -.09 \\
\hline \multirow[t]{3}{*}{ Gaze duration } & Intercept & 254.77 & 6.09 & 41.86 \\
\hline & Synonymous vs. Incongruent & 1.42 & 2.16 & .66 \\
\hline & Emoji vs. No Emoji & .93 & 1.23 & .76 \\
\hline \multirow[t]{3}{*}{ Total time } & Intercept & 381.83 & 15.19 & 25.14 \\
\hline & Synonymous vs. Incongruent & 7.09 & 4.72 & 1.50 \\
\hline & Emoji vs. No Emoji & 13.11 & 2.70 & 4.86 \\
\hline \multirow[t]{3}{*}{ Sentence reading time } & Intercept & $3,035.22$ & 124.30 & 24.42 \\
\hline & Synonymous vs. Incongruent & 115.27 & 23.35 & 4.94 \\
\hline & Emoji vs. No Emoji & -78.89 & 13.48 & -5.85 \\
\hline
\end{tabular}

Note. We contrasted the difference in processing between the synonymous and incongruent condition, as well as the difference in processing between the combined average processing time for synonymous and incongruent emoji conditions compared with the no-emoji condition by setting the intercept to the grand mean. The regression coefficients represent effect size in milliseconds. Negative $t$ s indicate a reduction in the effect where means for the incongruent condition were reduced compared with the synonymous condition, and means for the no-emoji condition were reduced compared with the emoji conditions. Significant effects are indicated in boldface

fixations; see Tables 3 and 4) were not significant. Nevertheless, the two later measures of processing (i.e., total time on the target word, sentence reading times) revealed significant effects (see Table 3). Specifically, the presence of an emoji (compared with its absence) led to greater skipping ( $z=$ $-2.13, p<.05)$ and shorter total time on the target word $(b=$ $13.11, t=4.86)$, but longer overall sentence reading times ( $b=$ $-78.89, t=-5.85$ ). Results of emoji presence replicate those of Cohn et al. (2018). Semantic processing of the emoji did not affect the probability of skipping or total time on the target word as the synonymous and incongruent contexts did not differ $(b=7.09, t=1.50)$. Nonetheless, overall sentence reading times were shorter when the emoji was synonymous compared with when it was incongruent $(b=115.27, t=4.96)$.

\section{Discussion}

To study the extent to which emojis are processed like words, we used eye tracking to document the time course of the

Table 4 Results of the linear mixed-effects models for the target word for the fixation probability measures (probability of single fixations, probability of skipping) as a function of emoji congruency (synonymous, incongruent, no emoji)

\begin{tabular}{|c|c|c|c|c|c|}
\hline Measure & Contrast & $b$ & SE & $z$ & $p$ \\
\hline \multirow[t]{3}{*}{ Probability of Single Fixations } & Intercept & 1.73 & .11 & 16.11 & $<.001$ \\
\hline & Synonymous vs. Incongruent & -.07 & .05 & -1.29 & .196 \\
\hline & Emoji vs. No Emoji & -.02 & .03 & -.78 & .436 \\
\hline \multirow[t]{3}{*}{ Probability of Skipping } & Intercept & -1.83 & .13 & -14.38 & $<.001$ \\
\hline & Synonymous vs. Incongruent & .01 & .05 & .14 & .890 \\
\hline & Emoji vs. No Emoji & -.06 & .03 & -2.13 & $<.05$ \\
\hline
\end{tabular}

Note. We contrasted the difference in processing between the synonymous and incongruent condition, as well as the difference in processing between the combined average processing of synonymous and incongruent emoji conditions compared with the no-emoji condition by setting the intercept to the grand mean. The regression coefficients represent effect size in log-odds space. Negative zs indicate a reduction in the effect, where log odds for the incongruent condition were reduced compared with the synonymous condition and log odds for the no-emoji condition were reduced compared with the emoji conditions. Significant effects are indicated in boldface 
semantic processing of emojis during natural sentence reading. Although a large prior literature has used eye tracking (Rayner, 1998, 2009) and ERPs (e.g., Dimigen et al., 2012; Kutas \& Hillyard, 1980) to explore the time course of semantic processing for words, our study provides one of the first demonstrations that the time course of semantic congruency effects on eye movements for emojis is analogous to effects that were previously shown for words. Specifically, the effect of congruency on emoji processing emerged in both early and late eye tracking measures. Relative to the synonymous emojis, the incongruent emojis elicited longer fixation durations and reductions in skipping and the probability of single-fixations. By providing finegrained time-course information, our study extends prior work that used self-paced reading paradigms and RSVP paradigms to investigate the semantic processing of emojis (Cohn et al., 2018; Weissman, 2019). Taken together, our results indicate that emojis show a timeline of semantic processing similar to words.

To investigate how emojis affect reading, we also contrasted the "synonymous" and "incongruent" emoji conditions with the "no-emoji" condition. Replicating Cohn et al. (2018), the presence of an emoji, relative to its absence, lengthened sentence reading times. Skipping rates on the target word increased in the presence of the emoji relative to its absence suggesting that attention is prioritized toward the emoji. In addition, total time on the target word decreased in the presence of an emoji, which potentially indicates that emojis can facilitate the postlexical integration of preceding text. Building on these findings, future work could further explore how verbal elements (i.e., text) and nonverbal elements (i.e., emojis) interact to facilitate reading. The nonverbal information provided by emojis is analogous to gestures (e.g., Feldman, Aragon, Chen, \& Kroll, 2017; McCulloch \& Gawne, 2018), which are known to enhance communication (Goldin-Meadow \& Alibali, 2013), but emojis could serve many functions by enhancing reading comprehension (Walther \& D'Addario, 2001), memory for text (Halvorson \& Hilverman, 2018), emotion (Riordan, 2017a, 2017b), irony, and humor comprehension (Dresner \& Herring, 2010; Weissman \& Tanner, 2018). Finally, as with gestures, emojis can exhibit different semantic relationships with the words that accompany them (Na'aman, Provenza, \& Montoya, 2017), such as the contrasting contribution of face and object emojis (Barach, Srinivasan, Fernandes, Feldman, \& Shaikh, 2020). Building off of Cohn (2016), future work could examine how these different relationships influence the interaction of verbal and nonverbal processing.

Regarding their theoretical implications, our results provide empirical constraints for models of eye movement control during reading. By showing a rapid time course of semantic congruency effects on the eye movement record for emojis, our results support the assumption that higher level lexical and linguistic factors can rapidly affect fixation durations during online reading (i.e., direct cognitive control; for a review of this debate, see Reingold et al., 2015). Also, contemporary models of eye movement control, including E-Z Reader and SWIFT, assume that reading entails a substantial amount of parafoveal processing, and there is growing evidence that semantic processing can begin in the parafovea during reading, as previously revealed by semantic preview effects (Hohenstein \& Kliegl, 2014; Rayner \& Schotter, 2014; Schotter, 2013; Schotter et al., 2015; Schotter \& Jia, 2016; Veldre \& Andrews, 2016; Yan et al., 2009; Yan et al., 2012; Yang et al., 2012). As evidence that the semantic processing of emojis also begins in the parafovea, here we show that semantic congruency modulates skipping rates for emojis. By demonstrating a time course of processing for emojis that overlaps with that of words, our results indicate that the time-course assumptions of current models of eye-movement control can potentially generalize to a novel condition that mixes a verbal element (i.e., the target word) with a spatially compact nonverbal element (i.e., an emoji). In essence, we identify a new experimental condition under which semantic similarity across verbal and nonverbal codes facilitates processing.

Our results may also clarify why semantic preview effects are easier to detect in the written form of some languages than of others. Emojis, like Chinese characters, are physically smaller than typical words written in alphabetic scripts. The Chinese script is logographic, and words are composed of characters that are not separated by spaces. Semantic preview effects are robust in Chinese, and they arise not only for single characters ( $\mathrm{Yu}$ et al., 2016) but also for their semantic sublexical components (Yan et al., 2009). The implication is that the spatial layout of a written word can influence the extent to which semantic parafoveal preview effects arise (for a related discussion, see Schotter et al., 2012).

In summary, emoji-word congruency effects provide an index of early semantic processing with implications for the control of saccades. One limitation of the present study is that the emoji was always located in its conventional position, at the end of the sentence with at least one intervening word between the emoji and its word counterpart. Therefore, future work would benefit from manipulating the spatial proximity of emoji and target word to further explore the time course of semantic processing. Most relevant is whether the parafoveal processing of emojis can affect the first-pass reading of preceding words (i.e., parafoveal-on-foveal effects; for a review, see Drieghe, 2011). Due to their spatially compact format, emojis are ideal for studying the time course of semantic processing, as well as the extent to which reading processes generalize over visual formats. Future work could use emojis to further explore a variety of reading variables, including word frequency and predictability. 


\section{Appendix}

Table 5 For each sentence the target word is presented in italics with the experimental target word to the left of the slash and the control target word to the right of the slash. The emojiswere presented at the end of the sentence and the synonymous emoji is presented to the left of the slash and the incongruent emoji is presented to the right of the slash

1 These homemade cookies/desserts are delicious $\because / 8$

2 Her little hamster/pet is adorable $8.8 /$ h

3 I'm so stressed, so I had a pizza/meal delivered to my dorm

4 My new sedan/ride was a good purchase

5 My tall coffee/beverage is just the right temperature - / ID

6 I prefer to weightlift/exercise before school 'I:

7 George had some bright socks/clothes on today /

8 When I'm hungry kiwi/fruit is a tasty and healthy choice $9 /{ }^{\circ}$

9 It's good to get popcorn/snacks for the movies III/ $/ 0$

10 Every week my brother flies/travels to New York City

11 I reached out to the new doctor/worker for advice

12 I don't like clowns/carnivals even though my sister loves them is: is

13 We screamed when the alien/monster jumped out at the haunted house

14 I bought the boys plastic swords/weapons to play with $\times / A_{2}$

15 The kids ran toward the rollercoaster/rides when we arrived

16 Don't forget to bring the hourglass/timer for charades tonight $\overline{3}$ (1)

17 I needed a candle/light during the power outage $1 /$

18 My teacher requires special pencils/supplies for this class

19 My picture needs more paint/color before its finished

20 It would be fun if we took a canoe/boat across the river $\phi /=$

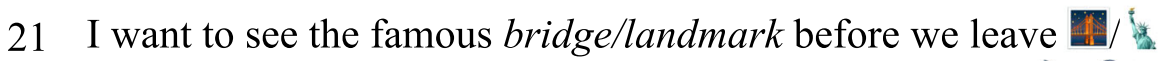

22 My dad picked up the wrench/tool that I left on the floor

23 I used all of my spoons/utensils so I'll have to clean them V ill

24 My family likes skiing/weekends at Hunter mountain

25 As a serious gymnast/athlete I have to practice every day $/ 0$

26 I had such good beef/meat last night

27 They forgot to bring the fries/side I ordered $1 /$ /

28 I bought the perfect diamond/accessory for myself at the mall $\nabla /$

29 I had to get that shot/medicine so I don't get sick

30 I really need chocolate/candy to satisfy my craving /@

31 Lucky for us we had wine/drinks at the boring party 1 /

32 I'm going to spend all my cash/savings on the trip [- [-

33 Please text/call me when you get to the mall

34 I wanted to look at the snakes/reptiles at the fair 2 
35 The kids have a project on lions/animals due next week / /

36 At the picnic caterpillars/insects were crawling on the table

37 My best friend received the trophy/award in the talent show $\frac{\text { I }}{2}$

38 I listen to piano/music to help me fall asleep $\mathrm{mI} / \mathbb{R}^{*}$

39 She is expecting the letter/mail to arrive by this afternoon

40 My aunt tried to give up sundaes/sweets for Lent this year $1 / 0$

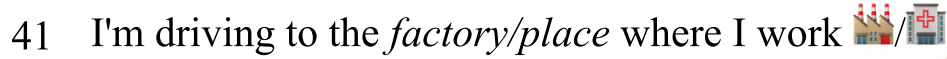

42 Shawn gave me roses/flowers for Valentine's Day /

43 I have to take care of the cactus/plant before vacation $4 / 2$

44 I love the stylish heels/shoes my mom let me borrow

45 I love the picture with the magical dragons/creatures at the gallery

46 I lost my suitcase/bag at the airport

47 There was a chilled shrimp/seafood platter at the buffet $5 /$ o

48 The event had many different types of whiskey/alcohol available

49 There was a terrified mouse/animal hiding under my desk

50 My friends and I like to see the frogs/wildlife at the pond

51 I needed a donut/treat after that hard test 0

52 I like watching soccer/sports at the bar across from campus $1 / 0$

53 Caroline grabbed the pumpkins/decorations this afternoon $\bigcirc /$ /

54 To keep us warm, we need some tents/shelter for the four of us

55 The young dancer/performer was excellent and totally worth seeing

56 I had the best pancakes/breakfast at the diner $\odot \mathbf{O}$.

57 My favorite beach/place is a two hour drive away /

58 I saw a brand new motorcycle/vehicle on the road this morning

59 I'm a fast typist/writer so my essay won't take long $\equiv / 2$

60 My roommate played her saxophone/song during the audition $\sqrt{6} / \&$

61 We should go to the fireworks/celebration tonight

62 As a competitive wrestler/athlete, my brother has a strict diet

63 You should've tasted the eggplant/vegetables Mike made for me

64 Jordan is studying owls/birds and their habitats

65 We were told by the police/staff that the street was closed $8 /{ }^{\circ}$

66 I tried not to cry at the wedding/ceremony for my cousin $2 /$

67 I need to use the shower/bathroom before I get into bed

68 We bought organic tomatoes/vegetables from the farmer's market

69 It is too windy/cold to walk to class today

70 Sharon made sure to bring cards/games to occupy the kids /

71 I'm going to design a robot/cool costume for Halloween

72 There is a spot close by to get sushi/food for takeout $\approx / 8$

73 My parents finally adopted a poodle/pet from the pound $3:<$

74 I will grab fresh strawberries/fruit at the grocery store / 
75 My friends wanted to see the gorilla/main exhibit at the zoo

76 My mom sent me spaghetti/dinner recipes that are quick and easy

77 We should spend some time surfing/outside tomorrow morning

78 It was the perfect day to wear my new shirt/outfit at school

79 There were so many bees/bugs near the park today

80 The news is broadcasting a tornado/weather update in the area $1 /$

81 I can't forget to grab the balloons/decorations for my mom $/$ at

82 I picked up my stylish sunglasses/accessories yesterday afternoon

83 We checked out the camera/electronics at Best Buy

84 I need some scissors/supplies for my office $9 / 0$

85 I think that chess/game is too hard for me

86 I found information about the castle/location before our tour $1 / 1 / 2$

87 Tonight there is this science/interesting demonstration near campus $/ 1$

88 I love the ring/gift my boyfriend got me $8 / 0$

89 I found a perfect puzzle/toy for my sister at the store

Table 6 Filler sentences and the accompanying comprehension question and answer to the comprehension question

\begin{tabular}{|c|c|c|c|}
\hline 1 & I'll order my chai with coconut milk and sugar & What was the emoji? & a coconut \\
\hline 2 & I can't wait to eat my sandwich at lunch $\downarrow$ & What was the emoji? & a sandwich \\
\hline 3 & If you want to laugh check out the goat videos on YouTube & What was the emoji? & a goat \\
\hline 4 & I stepped on a snail last night Q & What was the emoji? & a snail \\
\hline 5 & After our fifth date he finally kissed me goodnight & After the fifth date what happened? & a kiss goodnight \\
\hline 6 & I got my first paycheck on Friday & What came in on Friday? & the author's paycheck \\
\hline 7 & I knew I was smart when I got the only A on my history exam & What did the student know after the exam? & that he/she is smart \\
\hline 8 & I can't go since I have my haircut appointment & What appointment is this afternoon? & haircut \\
\hline 9 & I'm not a fan of bowling so count me out for tonight & What was the emoji? & bowling \\
\hline 10 & We got to use the measuring scales in the lab 1 & $\begin{array}{l}\text { What piece of equipment did they use in the } \\
\text { lab? }\end{array}$ & measuring scale \\
\hline 11 & That cracker was ridiculously stale & What was the emoji? & cheese \\
\hline 12 & Someone has to make cranberry sauce for Thanksgiving & What has to be made for Thanksgiving dinner? & cranberry sauce \\
\hline 13 & If I visit Africa I want to see a tiger on a safari tour & What was the emoji? & a koala Bear \\
\hline 14 & There's a great documentary about panda bears & What was the emoji? & a giraffe \\
\hline 15 & Karen couldn't help but laugh during that movie & What did Karen do during the movie? & laughed \\
\hline 16 & My new dream is to be a fashion designer for my career & What is the author's career goal? & fashion designer \\
\hline 17 & Sign up quick because the first drawing class is today & What is starting today? & drawing class \\
\hline 18 & I saw her sons bring in her couch through the door it & What were her sons carrying? & a couch \\
\hline 19 & Daniel left his wallet at home so he's running late & What was the emoji? & a key \\
\hline 20 & Carina picked up paper for the office $\not$ & What was the emoji? & a pushpin \\
\hline 21 & Don't forget to bring your water bottle to the library & $\begin{array}{l}\text { What shouldn't you forget to bring with you to } \\
\text { the library? }\end{array}$ & your water bottle \\
\hline 22 & For the hotdogs please grab some relish & What does the author need for the hotdogs? & relish \\
\hline 23 & I can make cupcakes for our visitors & What is being made for the visitors? & cupcakes \\
\hline 24 & My nutritionist told me to eat more dairy & What did the nutritionist say to have more of? & dairy \\
\hline 25 & The girl in the store tried to sell me lotion & What was the girl trying to sell? & lotion \\
\hline 26 & Meet me at the tennis courts for a match & Why are they meeting up? & to play tennis \\
\hline 27 & The little boy across the street always makes pig noises & What does the little boy do? & make pig noises \\
\hline 28 & Near Michelle's house there are cows that run loose & What is near Michelle's house? & cows \\
\hline 29 & I was so shocked when I tripped while climbing the stairs & How did the author feel after tripping? & shocked \\
\hline 30 & John's cousin is a professor at the University of Arizona & $\begin{array}{l}\text { What career does John's cousin have at the } \\
\text { University of Arizona? }\end{array}$ & Professor \\
\hline
\end{tabular}


Table 7 Means and summary of the results of the by-participant $\left(t_{1}\right)$ and by-item $\left(t_{2}\right)$ paired $t$ tests for the emoji for the fixation-based measures (first fixation, single fixation, gaze duration, total time, probability of single fixations, and probability of skipping) as well sentence reading times in sentences containing a target word that fit semantically with either emoji

\begin{tabular}{|c|c|c|c|c|}
\hline Measure & Synonymous emoji & Incongruent emoji & Mean difference & Emoji (synonymous, incongruent) \\
\hline \multirow[t]{2}{*}{ First fixation (ms) } & 306 & 319 & -12 & $t_{1}=-.88, p=.389, d=-.160$ \\
\hline & (11) & (17) & (14) & $t_{2}=-1.72, p=.089, d=-.182$ \\
\hline \multirow[t]{2}{*}{ Single fixation (ms) } & 321 & 332 & -11 & $t_{1}=-.66, p=.513, d=-.121$ \\
\hline & (13) & (19) & (16) & $T_{2}=-1.75, p=.084, d=-.185$ \\
\hline \multirow[t]{2}{*}{ Gaze duration (ms) } & 364 & 360 & 4 & $t_{1}=.23, p=.821, d=.042$ \\
\hline & (15) & (18) & (18) & $t_{2}=-.69, p=.489, d=-.074$ \\
\hline \multirow[t]{2}{*}{ Total time (ms) } & 407 & 401 & 6 & $t_{1}=.31, p=.760, d=.056$ \\
\hline & (19) & (19) & (18) & $t_{2}=-.74, p=.461, d=-.078$ \\
\hline \multirow[t]{2}{*}{ Probability of single fixations } & .86 & .88 & -.02 & $t_{1}=-.72 p=.479, d=-.131$ \\
\hline & $(.02)$ & $(.01)$ & $(.02)$ & $T_{2}=-1.24, p=.217, d=-.132$ \\
\hline \multirow[t]{2}{*}{ Probability of skipping } & .52 & .53 & -.01 & $t_{1}=-.33, p=.742, d=-.061$ \\
\hline & $(.03)$ & $(.03)$ & $(.02)$ & $T_{2}=-.32, p=.752, d=-.034$ \\
\hline \multirow[t]{2}{*}{ Sentence reading time (ms) } & 2,597 & 2,603 & -7 & $t_{1}=-.17, p=.868, d=-.031$ \\
\hline & $(125)$ & $(126)$ & $(41)$ & $t_{2}=-.19, p=.853, d=-.020$ \\
\hline
\end{tabular}

Note. For the paired $t$ tests, $d f$ for $t_{l}=(29), t_{2}=(88)$. Means in the table are from the by-participant analyses and standard error of the means are listed in parentheses (please see text for details)

Table 8 Summary of linear mixed-effects models random effects structures for the emoji LMMs and the target word LMMs

\begin{tabular}{llr}
\hline Measure & \multicolumn{2}{l}{ Random effects structure } \\
\cline { 2 - 3 } & Emoji LMM & Target word LMM \\
\hline First-fixation duration & $(1 \mid$ subjects $)+(1 \mid$ items $)$ & $(1 \mid$ subjects $)+(1 \mid$ items $)$ \\
Single-fixation duration & $(1 \mid$ subjects $)+(1 \mid$ items $)$ & $(1 \mid$ subjects $)+(1 \mid$ items $)$ \\
Gaze duration & $(1 \mid$ subjects $)+(1 \mid$ items $)$ & $(1 \mid$ subjects $)+(1 \mid$ items $)$ \\
Total time & $(1 \mid$ subjects $)+(1 \mid$ items $)$ & $(1 \mid$ subjects $)+(1 \mid$ items $)$ \\
Probability of single fixations & $(1 \mid$ subjects $)$ & $(1 \mid$ subjects $)+(1 \mid$ items $)$ \\
Probability of skipping & $(1 \mid$ subjects $)+(1 \mid$ items $)$ & $(1 \mid$ subjects $)+(1 \mid$ items $)$ \\
Sentence reading time & - & $(1 \mid$ subjects $)+(1 \mid$ items $)$
\end{tabular}

Note. All models were first fit using the maximal random effects structure (Barr et al., 2013) was fitted. However, for all models, the maximal random effects structure failed to converge, so each model was systematically trimmed until it converged, by removing correlations between random effects, and then random effects associated with the smallest variance. Once models converged, we used restricted maximum likelihood (REML) estimates for model comparisons involving correlation parameters or variance components (see text for details)

\section{References}

Barach, E., Srinivasan, V., Fernandes, R. B., Feldman, L. B., \& Shaikh, S. (2020). It's not just what you tweet, it's how you tweet it. Paper presented at the 7th European Conference on Social Media, Larnaca, Cyprus.

Barr, D. J., Levy, R., Scheepers, C., Tily, H. J. (2013) Random effects structure for confirmatory hypothesis testing: Keep it maximal. Journal of Memory and Language 68(3):255-278

Bates, D., Mächler, M., Bolker, B., \& Walker, S. (2015). Fitting linear mixed-effects models using lme4. ArXiv. https://doi.org/10.18637/ jss.v067.i0

Brysbaert, M., \& New, B. (2009). Moving beyond Kučera and Francis: A critical evaluation of current word frequency norms and the introduction of a new and improved word frequency measure for American English. Behavior Research Methods, 41(4), 977-990. https://doi.org/10.3758/BRM.41.4.977
Cohn, N. (2016). A multimodal parallel architecture: A cognitive framework for multimodal interactions. Cognition, 146, 304-323. https:// doi.org/10.1016/j.cognition.2015.10.007

Cohn, N., Roijackers, T., Schaap, R., \& Engelen, J. (2018). Are emoji a poor substitute for words? Sentence processing with emoji substitutions. Proceedings of the 40th Annual Conference of the Cognitive Science Society (pp. 15241529). Cognitive Science Society. http://www. cognitivesciencesociety.org/conference/cogsci-2018/

Cramer, H., de Juan, P., \& Tetreault, J. (2016). Sender-intended functions of emojis in US messaging. MobileHCI '16: Proceedings of the 18th International Conference on Human-Computer Interaction with Mobile Devices and Services (pp. 504-509). ACM. https://doi.org/ $10.1145 / 2935334.2935370$

Dimigen, O., Kliegl, R., \& Sommer, W. (2012). Trans-saccadic parafoveal preview benefits in fluent reading: A study with 
fixation-related brain potentials. NeuroImage, 62(1), 381-393. https://doi.org/10.1016/j.neuroimage.2012.04.006

Dresner, E., \& Herring, S. C. (2010). Functions of the nonverbal in CMC: Emoticons and illocutionary force. Communication Theory, 20(3), 249-268. https://doi.org/10.1111/j.1468-2885.2010.01362.x

Drieghe, D. (2011). Parafoveal-on-foveal effects on eye movements during reading. In S. P. Liversedge, I. D. Gilchrist, \& S. Everling (Eds.), The Oxford handbook of eye movements (pp. 839-855). Oxford: Oxford University Press.

Engbert, R., Longtin, A., \& Kliegl, R. (2002). A dynamical model of saccade generation in reading based on spatially distributed lexical processing. Vision Research, 42(5), 621-636. https://doi.org/10. 1016/S0042-6989(01)00301-7

Engbert, R., Nuthmann, A., Richter, E. M., \& Kliegl, R. (2005). SWIFT: A dynamical model of saccade generation during reading. Psychological Review, 112(4), 777-813. https://doi.org/10.1037/ 0033-295X.112.4.777

Feldman, L. B., Aragon, C., Chen, N. C., \& Kroll, J. F. (2017). Emoticons in text may function like gestures in spoken or signed communication. Behavioral and Brain Sciences, 40(3). https://doi.org/10.1017/ S0140525X15002903

Goldin-Meadow, S., \& Alibali, M. W. (2013). Gesture's role in speaking, learning, and creating language. Annual Review of Psychology, 64(1), 257-283. https://doi.org/10.1146/annurev-psych-113011143802

Gustafsson, V. (2017). Replacing words with emojis and its effect on reading time. [Paper presentation]. Umeå's 21 st Student Conference in Computing Science, Umeå, Sweden.

Halvorson, K. M., \& Hilverman, C. (2018, November 16). Emoji use influences memory for accompanying sentences [Poster]. Annual meeting of the Psychonomic Society, New Orleans, LA, United States.

Hohenstein, S., \& Kliegl, R. (2014). Semantic preview benefit during reading. Journal of Experimental Psychology: Learning, Memory, and Cognition, 40(1), 166-190. https://doi.org/10.1037/a0033670

Kelly, R., \& Watts, L. (2015). Characterising the inventive appropriation of emoji as relationally meaningful in mediated close personal relationships [Paper presentation]. Experiences of Technology Appropriation: Unanticipated Users, Usage, Circumstances, and Design, Oslo, Norway.

Kutas, M., \& Hillyard, S. A. (1980). Reading senseless sentences: Brain potentials reflect semantic incongruity. Science, 207, 203-205.

Lo, S. K. (2008). The Nonverbal communication functions of emoticons in computer-mediated communication. CyberPsychology \& Behavior, 11(5), 595-597. https://doi.org/10.1089/cpb.2007.0132

McCulloch, G., \& Gawne, L. (2018, June 25). Emoji grammar as beat gestures [Paper presentation]. First International Workshop on Emoji Understanding and Applications in Social Media, Stanford, CA, United States.

Na'aman, N., Provenza, H., \& Montoya, O. (2017). Varying linguistic purposes of emoji in (Twitter) context. Proceedings of ACL 2017, Student Research Workshop (pp. 136-141). https://doi.org/10. 18653/v1/P17-3022

Novak, P. K., Smailović, J., Sluban, B., \& Mozetič, I. (2015). Sentiment of Emojis. PLOS ONE, 10(12), Article e0144296. https://doi.org/10. 1371/journal.pone.0144296

Penolazzi, B., Hauk, O., \& Pulvermüller, F. (2007). Early semantic context integration and lexical access as revealed by event-related brain potentials. Biological Psychology, 74(3), 374-388. https://doi.org/ 10.1016/j.biopsycho.2006.09.008

Rayner, K. (1975). The perceptual span and peripheral cues in reading. Cognitive Psychology, 7(1), 65-81. https://doi.org/10.1016/00100285(75)90005-5

Rayner, K. (1998). Eye movements in reading and information processing: 20 Years of research. Psychological Bulletin, 124(3), 372-422. https://doi.org/10.1037/0033-2909.124.3.372
Rayner, K. (2009). Eye movements and attention in reading, scene perception, and visual search. The Quarterly Journal of Experimental Psychology, 62(8), 1457-1506. https://doi.org/10.1080/ 17470210902816461

Rayner, K., \& Schotter, E. R. (2014). Semantic preview benefit in reading English: The effect of initial letter capitalization. Journal of Experimental Psychology: Human Perception and Performance, 40(4), 1617-1628. https://doi.org/10.1037/a0036763

Reichle, E. D., Pollatsek, A., Fisher, D. L., \& Rayner, K. (1998). Toward a model of eye movement control in reading. Psychological Review, 105(1), 125-157. https://doi.org/10.1037/0033-295x.105.1.125

Reichle, E. D., Pollatsek, A., \& Rayner, K. (2012). Using E-Z Reader to simulate eye movements in nonreading tasks: A unified framework for understanding the eye-mind link. Psychological Review, 119(1), 155-185 https://doi.org/10.1037/a0026473

Reingold, E. M., Sheridan, H., \& Reichle, E. D. (2015). Direct lexical and nonlexical control of fixation duration in reading. In A. Pollatsek \& R. Treiman (Eds.), The Oxford handbook of reading (pp. 261-276). Oxford: Oxford University Press. https://doi.org/10.1093/oxfordhb/ 9780199324576.013 .10

Riordan, M. A. (2017a). Emojis as tools for emotion work: Communicating affect in text messages. Journal of Language and Social Psychology, 36(5), 549-567. https://doi.org/10.1177/ $0261927 X 17704238$

Riordan, M. A. (2017b). The communicative role of non-face emojis: Affect and disambiguation. Computers in Human Behavior, 76 , 75-86. https://doi.org/10.1016/j.chb.2017.07.009

Schotter, E. R. (2013). Synonyms provide semantic preview benefit in English. Journal of Memory and Language, 69(4), 619-633. https:// doi.org/10.1016/j.jml.2013.09.002

Schotter, E. R., Angele, B., \& Rayner, K. (2012). Parafoveal processing in reading. Attention, Perception, \& Psychophysics, 74(1), 5-35. https://doi.org/10.3758/s13414-011-0219-2

Schotter, E. R., \& Jia, A. (2016). Semantic and plausibility preview benefit effects in English: Evidence from eye movements. Journal of Experimental Psychology: Learning, Memory, and Cognition, 42(12), 1839-1866. https://doi.org/10.1037/xlm0000281

Schotter, E. R., Lee, M., Reiderman, M., \& Rayner, K. (2015). The effect of contextual constraint on parafoveal processing in reading. Journal of Memory and Language, 83, 118-139. https://doi.org/ 10.1016/j.jml.2015.04.005

Sereno, S. C., Brewer, C. C., \& O’Donnell, P. J. (2003). Context effects in word recognition: Evidence for early interactive processing. Psychological Science, 14(4), 328-333. https://doi.org/10.1111/ 1467-9280.14471

Tang, M., Chen, B., Zhao, X., \& Zhao, L. (2020). Processing network emojis in Chinese sentence context: An ERP study. Neuroscience Letters, 722, Article 134815. https://doi.org/10.1016/j.neulet.2020. 134815

Tigwell, G. W., \& Flatla, D. R. (2016). Oh that's what you meant!: Reducing emoji misunderstanding. Proceedings of the 18th International Conference on Human-Computer Interaction with Mobile Devices and Services Adjunct-MobileHCI '16 (pp. 859866). https://doi.org/10.1145/2957265.2961844

Veldre, A., \& Andrews, S. (2016). Is semantic preview benefit due to relatedness or plausibility? Journal of Experimental Psychology: Human Perception and Performance, 42(7), 939-952. https://doi. org/10.1037/xhp0000200

Veldre, A., \& Andrews, S. (2017). Parafoveal preview benefit in sentence reading: Independent effects of plausibility and orthographic relatedness. Psychonomic Bulletin \& Review, 24(2), 519-528. https:// doi.org/10.3758/s13423-016-1120-8

Walther, J. B., \& D'Addario, K. P. (2001). The impacts of emoticons on message interpretation in computer-mediated communication. Social Science Computer Review, 19(3), 324-347. https://doi.org/ 10.1177/089443930101900307 
Weissman, B. (2019). Emojis in sentence processing: An electrophysiological approach. WWW '19: Companion Proceedings of The 2019 World Wide Web Conference (pp. 478-479). https://doi.org/10. $1145 / 3308560.3316544$

Weissman, B., \& Tanner, D. (2018). A strong wink between verbal and emoji-based irony: How the brain processes ironic emojis during language comprehension. PLOS ONE, 13(8), e0201727. https:// doi.org/10.1371/journal.pone.0201727

Yan, M., Richter, E. M., Shu, H., \& Kliegl, R. (2009). Readers of Chinese extract semantic information from parafoveal words. Psychonomic Bulletin \& Review, 16(3), 561-566. https://doi.org/10.3758/PBR. 16.3.561

Yan, M., Zhou, W., Shu, H., \& Kliegl, R. (2012). Lexical and sublexical semantic preview benefits in Chinese reading. Journal of Experimental Psychology: Learning, Memory, and Cognition, 38(4), 1069-1075. https://doi.org/10.1037/a0026935

Yang, J., Wang, S., Tong, X., \& Rayner, K. (2012). Semantic and plausibility effects on preview benefit during eye fixations in Chinese reading. Reading and Writing, 25(5), 1031-1052. https://doi.org/10. 1007/s11145-010-9281-8

Yu, L., Cutter, M. G., Yan, G., Bai, X., Fu, Y., Drieghe, D., \& Liversedge, S. P. (2016). Word $n+2$ preview effects in threecharacter Chinese idioms and phrases. Language, Cognition and Neuroscience, 31(9), 1130-1149. https://doi.org/10.1080/ 23273798.2016 .1197954

Open practices statement

The materials are reported in the Appendix, and the authors can be contacted directly to receive access to the raw data.

Publisher's note Springer Nature remains neutral with regard to jurisdictional claims in published maps and institutional affiliations. 